\title{
Origin of thermal and non-thermal hard X-ray emission from the Galactic center
}

\author{
V.A. Dogiel ${ }^{* 1,2}$, K.S. Cheng ${ }^{3}$, D.O. Chernyshov ${ }^{2,3,4,5}$, H. Inoue ${ }^{1}$, C.-M. Ko ${ }^{5}$, M. \\ Kokubun $^{1}$, Y. Maeda ${ }^{1}$, K. Mitsuda ${ }^{1}$, and N.Y. Yamasaki ${ }^{1}$ \\ ${ }^{1}$ Institute of Space and Astronautical Science, 3-1-1, Yoshinodai, Sagamihara, Kanagawa, \\ 229-8510, Japan \\ ${ }^{2}$ I.E.Tamm Theoretical Physics Division of P.N.Lebedev Institute, Leninskii pr, 53, 119991 \\ Moscow, Russia \\ ${ }^{3}$ Department of Physics, University of Hong Kong, Pokfulam Road, Hong Kong, China \\ ${ }^{4}$ Moscow Institute of Physics and Technology, Institutskii lane, 141700 Moscow Region, \\ Dolgoprudnii, Russia \\ ${ }^{5}$ Institute of Astronomy, National Central University, Chung-Li 32054, Taiwan
}

\begin{abstract}
We analyse new results of CHANDRA and SUZAKU which found a flux of hard X-ray emission from the compact region around $\operatorname{Sgr} \mathrm{A}^{*}(\mathrm{r} \sim 100 \mathrm{pc})$. We propose that this emission is a consequence of a special transient accretion process when a part of captured star obtains an additional angular momentum. As a result a flux of subrelativistic protons is ejected from the Galactic black hole, which heats up the background plasma in the Galactic center up to temperature about 6-10 $\mathrm{keV}$ and produces by inverse bremsstrahlung a flux of non-thermal X-ray emission in the energy range above $10 \mathrm{keV}$.
\end{abstract}

7th INTEGRAL Workshop

September 8-11 2008

Copenhagen, Denmark

\footnotetext{
*Speaker.
} 


\section{Introduction}

The X-ray emission from the Galactic ridge in the energy range above $1 \mathrm{keV}$ was detected almost forty years ago [1] but its origin remains unknown till now. Two possibilities are debated, either this emission is really diffuse [2] or it is due to an accumulative effect of faint X-ray sources [3]. In the case of diffuse origin serious energetic problems arise if this emission is produced by thermal [4] or non-thermal [5-7]) bremsstrahlung of high energy electrons, since the required power of energy sources in the disk exceeds $10^{42} \mathrm{erg} \mathrm{s}^{-1}$, i.e. the maximum power which can be supplied in the disk by supernova stars. However, this energy problem can be solved in the case if these electrons are is-situ accelerated. If particles are accelerated from a background plasma, then the Maxwellian part of the spectrum is strongly distorted that mimics either a multi-temperature thermal emission or appearance of "a nonthermal tail". Less power is necessary for bremsstrahlung radiation in the case if it is generated by particles from the distorted Maxwellian part of the spectrum. Attempts to estimate the power of emitting particles in frames of pure thermal or pure non-thermal models may lead to incorrect values $[8,9]$.

Recently, essential arguments in favor of the idea that the Galactic ridge emission was due to cumulative emission of faint discrete X-ray sources was developed by Revnivtsev et al. (2006) [10]. Though this interpretation is not completely proved at present, it appears to be plausible.

Quite different situation may be in the Galactic center. This region is known to be peculiar in many respects. Therefore, the origin of X-ray emission from there may be somewhat different from the rest of the Galaxy. Though observations shows there a significant contribution of discrete sources which apply from $20 \%$ to $40 \%$ of the total flux (see [11, 12]), the SUZAKU data do not show correlation between the X-ray source distribution derived from Chandra data and the distribution of $6.7 \mathrm{keV}$ line [13] that may contradict the source origin of hard X-ray emission from the Galactic center. Observations show also essential distinctions between X-ray emission from the Galactic ridge and from the Galactic center. The Galactic center emission is seen as a completely separated spherical region around Sgr-A* whose radius is about $100-150$ pc [11, 14]. From the SUZAKU data it was derived that the ratio of 6.9 to $6.7 \mathrm{keV}$ iron lines (which traces the plasma temperature) is higher in the Galactic center than in the Ridge where this ratio is almost constant along the plane [15]. That may be a problem for the point-source interpretation, though one can assume that the source composition in the Galactic center differs from that in the disk.

The problem of the diffuse interpretation is that there are no evident sources of energy in the Galactic center which could provide plasma heating there. Thus, CHANDRA resolved only a weak X-ray point source at the position of supermassive black hole, Sgr A*, with a flux $L_{x} \sim 10^{33} \mathrm{erg}$ $\mathrm{s}^{-1}$ (see [16]). One likely scenario is that the Galactic nucleus was brighter in the past, possibly caused by a surge accretion onto the massive back hole.

Below we present a model of thermal and non-thermal hard X-ray emission from the Galactic center which is supposed to be due to specific processes of accretion on the central black hole.

\section{Parameters of X-ray Emission from the Galactic Center}

The Galactic Center component of X-ray emission was discovered with the Ginga satellite [17]. The ASCA telescope [14] measured the X-ray spectrum of the inner $\sim 150 \mathrm{pc}$ and found a 
number of emission lines from highly ionized elements which are characteristics for a $8-10 \mathrm{keV}$ thermal plasma with the density $0.4 \mathrm{~cm}^{-3}$. The total flux in the whole energy band of ASCA (2 $10 \mathrm{keV}$ ) is $10^{37} \mathrm{erg} / \mathrm{s}$. This $8 \mathrm{keV}$ plasma would be too hot to be bound to the Galactic center and therefore would form a slow wind or fountain of plasma with an expansion velocity equal to the sound speed, $\sim 1600 \mathrm{~km} \mathrm{~s}^{-1}$. Then the energy required to sustain such a freely expanding plasma is about $\sim 10^{41}-10^{42} \mathrm{erg} \mathrm{s}^{-1}$. Recent CHANDRA observations [11] showed also an intensive X-ray emission at the energy $E_{x} \sim 8 \mathrm{keV}$ from the inner $20 \mathrm{pc}$ of the Galaxy. This emission is distributed spherically symmetric around Sgr A*. The plasma density was estimated in limits $0.1-0.2 \mathrm{~cm}^{-3}$. The power derived from the CHANDRA data which is required for the plasma heating is also higher than provided by SNs.

SUZAKU observations [13] found also clear evidence for a hot plasma in the galactic central region with the diameter about 20 acrminutes (i.e $\sim 50-60 \mathrm{pc}$ ). Using the $6.9 / 6.7 \mathrm{keV}$ ratio it was concluded that the spectrum in 5-11.5 keV range was naturally explained by a $6.5 \mathrm{keV}$-temperature plasma in collisional ionization equilibrium. The origin of the hot temperature plasma is unclear since as in the case of $6 \mathrm{keV}$ thermal emission there are no evident sources of energy in the Galactic central region.

SUZAKU data suggested also that the continuum flux from the Galactic central region contained an additional hard component. Up to recently any direct confirmation of nonthermal emission at energies above $10 \mathrm{keV}$ has been unavailable. New SUZAKU observations [18] performed in the range from 14 to $40 \mathrm{keV}$ showed a prominent hard $\mathrm{X}$-ray emission whose spectrum is power-law with the spectral index ranging from 1.8 to 2.5 . The total luminosity of the power-law component from the central region $\left(|l|<2^{\circ},|b|<0.5^{\circ}\right)$ is $(4 \pm 0.4) \times 10^{36} \mathrm{erg} \mathrm{s}^{-1}$. The spatial distribution of hard X-rays correlates with the distribution of hot plasma.

These spectra are consistently represented by a cutoff power-law model,

$$
f(E)=K(E / 1 \mathrm{keV})^{-\Gamma} \exp \left(-E / E_{c}\right)
$$

with $\Gamma$ and $E_{c}$ varying from region to region over $1.2-2.2$ and $19-50 \mathrm{keV}$, respectively.

The origin of the hard X-ray emission is also unclear.

\section{Energy Release in the Galactic Center}

We suppose that the necessary energy for X-ray emission can be supplied by active processes near the Galactic center, which is expected to be a region of energy release due to accretion on the central supermassive black hole. The frequency of star capture by supermassive black holes ranges from $10^{-5}$ to $10^{-4}$ years $^{-1}$ [20-22]. Though the central black hole does not show high activity at present, it is likely that it was active in the past.

Processes of star accretion generate fluxes of high energy particles which are seen as jets of relativistic leptons or hadrons. In the last case secondary positrons are produced by hadron collisions that may be a source of the annihilation emission from the Galactic bulge (see [19]). Whether relativistic protons are accelerated near black hole, it is questionable, but star accretion processes evidently produce fluxes of subrelativistic protons.

As it was shown by Ayal et al. (2000) [21] once passing the pericenter, the star is tidally disrupted into a very long and dilute gas stream. Approximately $75 \%$ of the star was not accreted 
but instead became unbounded. This unbounded mass will receive additional angular momentum and the average energy of unbounded particles is about $E_{\text {esc }} \sim 100 \mathrm{MeV}$ nucleon $^{-1}$.

We approximate the energy distribution of these escaped nuclei as

$$
Q_{0}^{e s c}=\bar{N} \delta\left(E-E_{e s c}\right),
$$

where $\bar{N}$ is total amount of particles ejected during stellar capture.

For a single capture of one solar mass star we estimate the total number of unbounded particles as $\bar{N} \simeq 10^{57}$ protons and the total energy in unbounded protons about $W \simeq 10^{53} \mathrm{erg}$. For the star capture frequency $v \simeq 10^{-5} \div 10^{-4}$ year $^{-1}$ it gives the average energy input about $\dot{\mathrm{W}} \simeq 10^{41} \div$ $10^{42} \mathrm{erg} \mathrm{s}^{-1}$.

Processes of particle distribution in the Galactic central region are questionable since we do not know much about medium conditions there. However, simple estimates from [23] and [24] for the Galactic central region give the value, $D \sim 10^{27} \mathrm{~cm}^{2} \mathrm{~s}^{-1}$ that is close to the average cosmic ray coefficient of diffusion in the Galaxy (see [25]).

For the $1^{\circ} \div 2^{\circ}$ radius central region we take the temperature $6.5 \mathrm{keV}$ and the density of this gas $n \simeq 0.1 \div 0.4 \mathrm{~cm}^{-3}$ as it follows from observations $[11,14,13]$.

Then the time-dependent spectrum of subrelativistic protons, $N(\mathbf{r}, E, t)$, in the central $1^{\circ} \times 1^{\circ}$ region injected from accretion processes can be calculated from the equation

$$
\frac{\partial N}{\partial t}-\nabla D \nabla N+\frac{\partial}{\partial E}(b(E) N)=Q(E, t)
$$

where $d E / d t \equiv b(E)$ is the rate of proton energy losses. Subrelativistic protons lose their energy by Coulomb collisions. The injection of protons by accretion processes can be described by $Q(E, \mathbf{r}, t)=\sum_{k=0} Q_{k}(E) \delta\left(t-t_{k}\right) \delta(\mathbf{r})$, where $t_{k}$ is the injection time, and the functions $Q_{k}(E)$ are given by Eq. (3.1). Suppose $T$ is the average time between two capture events, then $t_{k}=k \times T$.

The solution of Eq. (3.2) is (see [26])

$$
N(\mathbf{r}, E, t)=\sum_{k=0} \int_{0}^{\infty} d E_{0} Q_{k}\left(E_{0}\right) G_{k}\left(\mathbf{r}, E, t ;, E_{0}\right)
$$

where

$$
G_{k}\left(\mathbf{r}, E, t ; E_{0}, t_{k}\right)=\frac{1}{|b(E)|} \frac{\delta\left(t-t_{k}-\tau\right)}{(4 \pi \lambda)^{3 / 2}} \exp \left[-\frac{\mathbf{r}^{2}}{4 \lambda}\right]
$$

and

$$
\tau\left(E, E_{0}\right)=\int_{E_{0}}^{E} \frac{d E}{b(E)} \quad \text { and } \quad \lambda=\int_{E_{0}}^{E} \frac{D(E)}{b(E)} d E
$$

\section{Energy Dissipation of Subrelativistic Protons and Plasma Heating}

As one can see from the solution (3.3) the density of emitted protons is almost stationary at distances $r \gg \sqrt{D T}$ but the number of these protons varies strongly in the central region $r \ll \sqrt{D T}$.

Subrelativistic protons loose their energy mainly by ionization or by heating of background plasma [27]. The rate of these energy losses is

$$
\frac{d E}{d t}=-\frac{4 \pi n e^{4} \ln \Lambda_{1}}{m_{e} \mathrm{v}_{p}}
$$


where $\mathrm{v}_{p}$ is the proton velocity, and $\ln \Lambda_{1}$ is the Coulomb logarithm. For the medium parameters the lifetime of $\leq 100 \mathrm{MeV}$ protons is about

$$
\tau_{l} \sim \int_{E_{\text {esc }}}^{E_{t h}} \frac{d E}{b(E)} \sim 2 \cdot 10^{14} \mathrm{~s}
$$

Where $E_{\text {esc }} \sim 100 \mathrm{MeV}$ is the injection energy of protons and $E_{t h}$ is the energy of thermal plasma. For this time protons fill the region around the Galactic center of the radius $r \sim \sqrt{D \tau_{l}} \sim 150 \mathrm{pc}$.

According to [14] the temperature to which a plasma in a volume of the radius $R$ is heated by an external source with the power $Q$ can be estimated from

$$
\rho U_{s}(T) 4 \pi R^{2} T \simeq Q
$$

where $U_{s}$ is the sound speed, $\rho$ and $T$ are the plasma density and the temperature. Koyama et al. (1996) [14] concluded that the plasma in the $1^{\circ} \div 2^{\circ}$ radius central region can be heated up to the observed temperature $T \sim 6 \div 10 \mathrm{keV}$ if $Q \sim 10^{41} \div 10^{42} \mathrm{erg} \mathrm{s}^{-1}$. From our analysis it follows that just this energy dissipates in the central region due to energy losses of subrelativistic protons.

\section{Nonthermal Emission from the Galactic Center}

Interaction of subrelativistic protons with plasma results also in production of bremsstrahlung photons (inverse bremsstrahlung radiation). Though the rate of these energy losses is negligible in comparison with the above-mentioned Coulomb energy losses, nevertheless, these losses generate emission in the energy range higher than the thermal emission of background plasma and hence can be observed. Subrelativistic protons generate bremsstrahlung photons with characteristic energies about $E_{x}<\left(m_{e} / M\right) E_{p}$ where $E_{p}$ is the kinetic energy of protons and $m$ and $M$ are masses of an electron and a proton. For the proton energies $E \leq 100 \mathrm{MeV}$ the bremsstrahlung radiation is in the range $E_{x}<60 \mathrm{keV}$. The cross-section of inverse bremsstrahlung radiation is [27]

$$
\frac{d \sigma_{b r}}{d E_{x}}=\frac{8}{3} Z^{2} \frac{e^{2}}{\hbar c}\left(\frac{e^{2}}{m c^{2}}\right)^{2} \frac{m c^{2}}{E^{\prime}} \frac{1}{E_{x}} \times \ln \frac{\left(\sqrt{E^{\prime}}+\sqrt{E^{\prime}-E_{x}}\right)^{2}}{E_{x}} .
$$

Here $E^{\prime}=(m / M) E_{p}$. Then the intensity of inverse bremsstrahlung can be calculated from

$$
I_{\mathbf{I}}=\int_{\mathbf{l}} N_{p}(E, \mathbf{r}, t) \frac{d \sigma_{b r}}{d E_{x}} \mathrm{v}_{p} n d \mathbf{l}
$$

where the vector $\mathbf{l}$ determines the direction of observations.

The spatial distribution of this emission in the Galactic center as observed from Earth with the SUZAKU data from [18] is shown in Fig. 1. The energy spectrum of hard X-ray emission observed by SUZAKU from the central $30 \mathrm{pc}$ radius [18] is shown in Fig. 2. The calculated spatial distribution and the X-ray spectrum of inverse bremsstrahlung radiation in the same directions are shown by the solid lines. One can see nice coincidence between the data and calculations. We can easily estimate the total energy output of hard X-ray emission in the energy range $14-40$ $\mathrm{keV}$ produced by inverse bremsstrahlung. The estimated flux due to proton bremsstrahlung equals $4.3 \cdot 10^{36} \mathrm{erg} \mathrm{s}^{-1}$, that is about of the flux observed by SUZAKU from the Galactic center in the same energy range. 


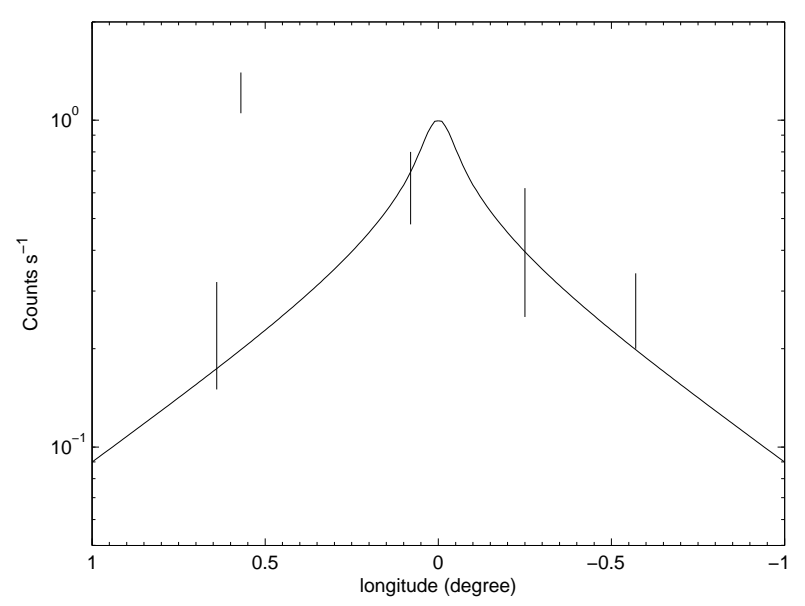

Figure 1: The spatial distribution of inverse bremsstrahlung emission with the SUZAKU data.

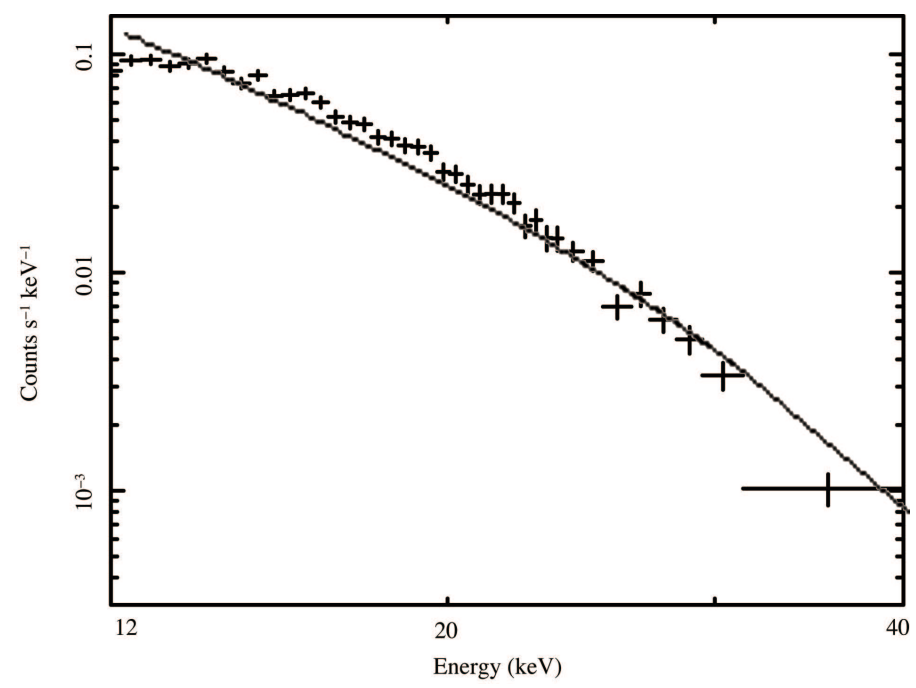

Figure 2: The spectrum of inverse bremsstrahlung emission generated by subrelativistic protons in the Galactic center region and the flux observed by SUZAKU from the Galactic central region. For the convolution of tour theoretical results to the SUZAKU data we used the average effective area of the SUZAKU telescope equaled $\sim 150 \mathrm{~cm}^{2}$ and the detection efficiency of photons to be $\sim 70 \%$

\section{Conclusion}

Subrelativistic protons are naturally generated by star captures nearby massive black holes. For one solar mass stars the energy of these protons is about $100 \mathrm{MeV}$ and the total energy release in protons is about $\sim 10^{41} \mathrm{erg} \mathrm{s}^{-1}$. This energy is mainly transferred into plasma heating. The size of heating area is estimated to be $\sim 150$ pc (i.e. $\sim 1^{\circ}$ ). For the estimated plasma escape time $\sim 50000$ years $[11,14]$ the background plasma should be heated up to the temperature $6-8 \mathrm{keV}$ just as observed. Subrelativistic protons loose also their energy by inverse bremsstrahlung. The rate of 
these losses is several orders of magnitude less than that of ionization, nevertheless the total energy flux of this emission is about $4 \cdot 10^{36} \mathrm{erg} \mathrm{s}^{-1}$ in the energy range $E_{x}<60 \mathrm{keV}$. that explains well the data obtained by SUZAKU in the range $14-40 \mathrm{keV}$.

\section{Acknowledgments}

VAD and DOC were partly supported by the RFBR grant 08-02-00170-a, the NSC-RFBR Joint Research Project No 95WFA0700088 and by the grant of a President of the Russian Federation "Scientific School of Academician V.L.Ginzburg". KSC is supported by a RGC grant of Hong Kong Government under HKU 7014/07P.

\section{References}

[1] Bleach, R. D., Boldt, E. A., Holt, S. S.,Schwartz, D. A., \& Serlemitsos, P. J., X-Ray Emission from the Galactic Disk, ApJL 174, (1972), 101

[2] Ebisawa, K., Tsujimoto, M., Paizis, A., Hamaguchi, K., Bamba, A., Cutri, R., Kaneda, H., Maeda, Y., Sato, G., Senda, A., Ueno, M., Yamauchi, S., Beckmann, V., Courvoisier, T. J.-L., Dubath, P., \& Nishihara, E., Chandra Deep X-Ray Observation of a Typical Galactic Plane Region and Near-Infrared Identification, ApJ 635, (2005), 214

[3] Krivonos, R., Revnivtsev, M., Churazov, E., Sazonov, S., Grebenev, S., \& Sunyaev, R., Hard X-ray emission from the Galactic ridge, A\&A 463, (2007), 957

[4] Inoue, H., Highlights of X-Ray Astronomy Mainly Based on ASCA Results, PThPS 143, (2001), 99I

[5] Skibo J.G., Ramaty R., \& Purcell W.R., Implications of the diffuse galactic continuum, A\&AS 120 (1996), 403

[6] Valinia A., Tatischeff V., Arnaud K., Ebisawa K., \& Ramaty R., On the Origin of the Iron K Line in the Spectrum of The Galactic X-Ray Background, ApJ 543, (2000), 733

[7] Dogiel, V. A., Schönfelder, V., \& Strong, A. W., Nonthermal hard X-ray emission from the Galactic Ridge, A\&A 382, (2002), 730

[8] Dogiel, V.A., Inoue, H., Masai, K., Schönfelder, V., \& Strong, A.W., The Origin of Diffuse X-Ray Emission from the Galactic Ridge. I. Energy Output of Particle Sources, ApJ 581, (2002), 1061

[9] Dogiel, V. A., Colafrancesco, S., Ko, C. M., Kuo, P. H., Hwang, C. Y., Ip, W. H., Birkinshaw, M., \& Prokhorov, D. A., In-situ acceleration of subrelativistic electrons in the Coma halo and the halo's influence on the Sunyaev-Zeldovich effect, A\&A 461, (2007),433

[10] Revnivtsev, M., Sazonov, S., Gilfanov, M., Churazov, E., \& Sunyaev, R., Origin of the Galactic ridge $X$-ray emission, $A \& A$ 452, (2006), 169

[11] Muno, M. P., Arabadjis, J. S., Baganoff, F. K., Bautz, M. W., Brandt, W. N., Broos, P. S., Feigelson, E. D., Garmire, G. P., Morris, M. R., \& Ricker, G. R., The Spectra and Variability of X-Ray Sources in a Deep Chandra Observation of the Galactic Center, ApJ 613, (2004), 1179

[12] Revnivtsev, M., Vikhlinin, A., \& Sazonov, S., Resolving the Galactic ridge X-ray background, A\&A 473, (2007), 857 
[13] Koyama, K., Hyodo, Y., Inui, T., Nakajima, H., Matsumoto, H., Tsuru, T. G., Takahashi, T., Maeda, Y., Yamazaki, N. Y., Murakami, H., Yamauchi, S., Tsuboi, Y., Senda, A., Kataoka, J., Takahashi, H., Holt, S. S., \& Brown, G. V., Iron and Nickel Line Diagnostics for the Galactic Center Diffuse Emission, PASJ 59, (2007), 245

[14] Koyama, K., Maeda, Y., Sonobe, T., Takeshima, T., Tanaka, Y., \& Yamauchi, S., ASCA View of Our Galactic Center: Remains of Past Activities in X-Rays?, PASJ 48, (1996), 249

[15] Yamauchi, S., Ebisawa, K., Tanaka, Y., Koyama, K., Matsumoto, H., Yamasaki, N. Y., Takahashi, H., \& Ezoe, Y., Iron Emission Lines on the Galactic Ridge Observed with Suzaku, astro-ph/0810.0317

[16] Baganoff, F. K., Maeda, Y., Morris, M., Bautz, M. W., Brandt, W. N., Cui, W., Doty, J. P., Feigelson, E. D., Garmire, G. P., Pravdo, S. H., Ricker, G. R., \& Townsley, L. K., Chandra X-Ray Spectroscopic Imaging of Sagittarius $A^{*}$ and the Central Parsec of the Galaxy, ApJ 591, (2003), 891

[17] Koyama, K., Awaki, H., Kunieda, H., Takano, S., \& Tawara, Y., Intense 6.7-keV iron line emission from the Galactic Centre, Nature 339, (1989), 603

[18] Yuasa, T., Tamura, K., Nakazawa, K., Kokubun, M., Makishima, K., Bamba, A., Maeda, Y., Takahashi, T., Ebisawa, K., Senda, A., Hyodo, Y., Tsuru, T. G., Koyama, K., Yamauchi, S., \& Takahashi, H., Suzaku Detection of Extended/Diffuse Hard X-Ray Emission from the Galactic Center, PASJ 60, (2008), 207

[19] Cheng, K. S., Chernyshov, D. O., \& Dogiel, V. A., Diffuse gamma-ray emission from the Galactic center - a multiple energy injection model, A\&A, 473, (2007), 351

[20] Syer, D., \& Ulmer, A., Tidal disruption rates of stars in observed galaxies, MNRAS 306, (1999), 35

[21] Ayal, S., Livio, M., \& Piran, T., Tidal Disruption of a Solar-Type Star by a Supermassive Black Hole, Large-Amplitude X-Ray Outbursts from Galactic Nuclei: A Systematic Survey using ROSAT Archival Data, ApJ 545, (2000), 772

[22] Donley, J. L., Brandt, W. N., Eracleous, M., \& Boller, Th., Large-Amplitude X-Ray Outbursts from Galactic Nuclei: A Systematic Survey using ROSAT Archival Data, ApJ 124, (2002), 1308

[23] LaRosa, T. N., Lazio, T. Joseph W., \& Kassim, N. E., A New System of Parallel Isolated Nonthermal Filaments near the Galactic Center: Evidence for a Local Magnetic Field Gradient, ApJL 563, (2001), 163

[24] Jean, P., Knödlseder, J., Gillard, W., Guessoum, N., Ferriere, K., Marcowith, A., Lonjou, V., \& Roques, J. P., Spectral analysis of the Galactic e+e- annihilation emission, A\&A 445, (2006), 579

[25] Berezinskii V.S., Bulanov S.V., Dogiel V.A., Ginzburg V.L., \& Ptuskin V.S., Astrophysics of Cosmic Rays, (ed. V.L.Ginzburg, North-Holland, Amsterdam, 1990)

[26] Syrovatskii S.I., The Distribution of Relativistic Electrons in the Galaxy and the Spectrum of Synchrotron Radio Emission, Sov.Astron. 3, (1959), 22

[27] S. Hayakawa: Cosmic Ray Physics (Wiley-Interscience, 1969) 